\title{
Abundant Lump Solutions and Interaction Phenomena to the Kadomtsev-Petviashvili- Benjamin-Bona-Mahony Equation
}

\author{
Jianqing Lü, Sudao Bilige*, Xiaoqing Gao, Yuexing Bai, Runfa Zhang \\ College of Sciences, Inner Mongolia University of Technology, Hohhote, China \\ Email: *inmathematica@126.com
}

How to cite this paper: Lü, J.Q., Bilige, S., Gao, X.Q., Bai, Y.X. and Zhang, R.F. (2018) Abundant Lump Solutions and Interaction Phenomena to the Kadomtsev-PetviashviliBenjamin-Bona-Mahony Equation. Journal of Applied Mathematics and Physics, 6, 1733-1747.

https://doi.org/10.4236/jamp.2018.68148

Received: July 26, 2018

Accepted: August 26, 2018

Published: August 29, 2018

Copyright $\odot 2018$ by authors and Scientific Research Publishing Inc. This work is licensed under the Creative Commons Attribution International License (CC BY 4.0).

http://creativecommons.org/licenses/by/4.0/

\begin{abstract}
In this paper, we obtained a kind of lump solutions of the KadomtsevPetviashvili-Benjamin-Bona-Mahony (KP-BBM) equation with the assistance of Mathematica. Some contour plots with different determinant values are sequentially made to show that the corresponding lump solutions tend to zero when $x^{2}+y^{2} \rightarrow \infty$. Particularly, lump solutions with specific values of the include parameters are plotted, as illustrative examples. Finally, a combination of stripe soliton and lump soliton is discussed to the KP-BBM equation, in which such a solution presents two different interesting phenomena: lump-kink and lump-soliton. Simultaneously, breather rational soliton solutions are displayed.
\end{abstract}

\section{Keywords}

Lump Solution, KP-BBM Equation, Hirota Bilinear Form, Interaction Phenomenon, Breather Soliton

\section{Introduction}

Soliton, rogue waves, lump solutions, breather waves and interaction solutions of nonlinear evolution equations (NLEEs) have attracted more and more attention [1] [2] [3] [4], and lump solutions are a kind of rational function and localized in all directions of space. Lump-soliton solutions have the meromorphic structures which can guarantee their stability [5]. Furthermore, lump solutions can be regarded as the localized wave configurations which decay rationally to the asymptotic values, and lump solitons move with the uniform velocities [6]. Lump solution may not be able to maintain its amplitude and shape through the interaction of the soliton solution. This interaction is inelastic [7]. Lump solu- 
tions have been found for kinds of integrable equation [8] [9] [10]. Lump solutions emerge the non-linear patterns, such as optic media, plasma, shallow water wave, and Bose-Einstein condensate [11].

Recently Ma W. X. found out that the approach to solving the lump solutions of NLEEs use the Hirota bilinear form [12]. The study of lump solution has been lack of development because of the complexity in the process that the lump solution of NLEEs can be solved. He successfully proved form of the solution and its existence [13]. By using this method, some researchers perfectly constructed the lump solutions and the interaction solutions [14]-[38] and breather waves [11], [39] of NLEEs.

In the following, we consider the Kadomtsev-Petviashvili-Benjamin-Bona-Mahony (KP-BBM) equation

$$
u_{x t}+u_{x x}+\alpha\left(u^{2}\right)_{x x}+\beta u_{x x x t}+\gamma u_{y y}=0 .
$$

The KP-BBM equation is formulated using the KP equation, derived from the standard BBM equation [40]. Some of the previous studies have been done [41] [42] [43] [44].

In the present paper, we would like to focus on KP-BBM equation. It has a Hirota bilinear form, and so, we will do a search for the positive quadratic function solutions to the corresponding bilinear KP-BBM equation. Firstly, we will obtain five different classes of positive quadratic function solutions. Secondly, the interaction solution constructed through symbolic computations beginning with a linear combination ansatz, in which such solution presents two different interesting phenomena: lump-kink and lump-soliton. Thirdly, breather rational soliton solutions are derived. Finally, some conclusions will be drawn at the end of this article.

\section{Lump Solution}

Under the bilinear transformation

$$
u=2(\ln f)_{x x} .
$$

Equation (1) is turned into the following Hirota bilinear form:

$$
\begin{aligned}
& \operatorname{KP}-\operatorname{BBM}(f):\left(D_{x} D_{t}+D_{x}^{2}+\beta D_{x}^{3} D_{t}+\gamma D_{y}^{2}+\alpha D_{x}^{2}-3 \beta D_{x} D_{t}\right)(f \cdot f) \\
& =2\left[f f_{x t}-f_{t} f_{x}+f_{x x} f-f_{x}^{2}+\beta\left(f f_{x x x t}-3 f_{x} f_{x x t}+3 f_{x x} f_{x t}-f_{t} f_{x x x}\right)\right. \\
& \left.\quad+\gamma\left(f_{y y} f-f_{y}^{2}\right)+\alpha\left(f_{x x} f-f_{x}^{2}\right)-3 \beta\left(f_{x t} f-f_{t} f_{x}\right)\right]=0,
\end{aligned}
$$

where the operator $D$ is defined:

$$
\begin{aligned}
D_{t}^{l} D_{x}^{k} D_{y}^{n} f \cdot g= & \left(\frac{\partial}{\partial t}-\frac{\partial}{\partial t^{\prime}}\right)^{l}\left(\frac{\partial}{\partial x}-\frac{\partial}{\partial x^{\prime}}\right)^{k}\left(\frac{\partial}{\partial y}-\frac{\partial}{\partial y^{\prime}}\right)^{n} f(x, y, t) \\
& \cdot g\left(x^{\prime}, y^{\prime}, t^{\prime}\right) \mid x=x^{\prime}, y=y^{\prime}, t=t^{\prime} .
\end{aligned}
$$

To search for the quadratic function solution of the bilinear KP-BBM equation, we suppose 


$$
f=g^{2}+h^{2}+a_{9}, g=a_{1} x+a_{2} y+a_{3} t+a_{4}, h=a_{5} x+a_{6} y+a_{7} t+a_{8},
$$

where $a_{i}(i=1, \cdots, 9)$ are real parameters to be determined later. To figure out a set of algebraic equations in $a_{i}(i=1, \cdots, 9)$, we substitute (4) into (3) and equate all the coefficients of different polynomials of $x, y, t$ to zero. We obtain a set of algebraic equations in $a_{i}(i=1, \cdots, 9)$. Solving the set of algebraic equations, we can find the following sets of solutions.

Case 1:

$$
\begin{gathered}
a_{1}=\frac{a_{4} a_{5}}{a_{8}}, a_{2}=-\frac{a_{6} a_{8}}{a_{4}}, a_{3}=\frac{a_{4} a_{6} \alpha \sqrt{\gamma}}{\sqrt{3 \beta a_{4}^{2}(\alpha+3 \beta)}}, a_{4}=a_{4}, a_{5}=\frac{\sqrt{3} a_{6} a_{8} \sqrt{\beta} \sqrt{\gamma}}{\sqrt{a_{4}^{2}(\alpha+3 \beta)}}, \\
a_{6}=a_{6}, a_{7}=\frac{a_{6} a_{8} \alpha \sqrt{\gamma}}{\sqrt{3 a_{4}^{2} \beta(\alpha+3 \beta)}}, a_{8}=a_{8}, a_{9}=-\frac{9 a_{6}^{2}\left(a_{4}^{2}+a_{8}^{2}\right) \alpha \beta^{2} \gamma}{a_{4}^{2}(\alpha+3 \beta)^{2}},
\end{gathered}
$$

where $a_{4}, a_{6}, a_{7}, a_{8}$ are arbitrary constants, and all these constants are satisfied with the condition as follows:

$$
a_{4} a_{8} \beta(\alpha+3 \beta) \neq 0, \alpha \gamma<0 .
$$

This set leads to a kind of positive quadratic function solutions to the bilinear KP-BBM equation in (3)

$$
\begin{aligned}
f= & {\left[\frac{a_{4} a_{5}}{a_{8}} x-\frac{a_{6} a_{8}}{a_{4}} y+\frac{a_{4} a_{6} \alpha \sqrt{\gamma}}{\sqrt{3 \beta a_{4}^{2}(\alpha+3 \beta)}} t+a_{4}\right]^{2} } \\
& +\left[\frac{\sqrt{3} a_{6} a_{8} \sqrt{\beta} \sqrt{\gamma}}{\sqrt{a_{4}^{2}(\alpha+3 \beta)}} x+a_{6} y+\frac{a_{6} a_{8} \alpha \sqrt{\gamma}}{\sqrt{3 a_{4}^{2} \beta(\alpha+3 \beta)}} t+a_{8}\right]^{2} \\
& -\frac{9 a_{6}^{2}\left(a_{4}^{2}+a_{8}^{2}\right) \alpha \beta^{2} \gamma}{a_{4}^{2}(\alpha+3 \beta)^{2}}
\end{aligned}
$$

and the resulting is a kind of positive quadratic function solutions. In turn, we gain the lump solution to the KP-BBM Equation in (1) by using the transformation (2)

$$
u_{1}(x, y, t)=\frac{4\left(a_{1}^{2}+a_{5}^{2}\right) f-8\left(a_{1} g+a_{5} h\right)^{2}}{f^{2}},
$$

where the function $f$ is defined by (6), and the functions $g$ and $h$ are given as follows:

$$
\begin{aligned}
& g=\frac{a_{4} a_{5}}{a_{8}} x-\frac{a_{6} a_{8}}{a_{4}} y+\frac{a_{4} a_{6} \alpha \sqrt{\gamma}}{\sqrt{3 \beta a_{4}^{2}(\alpha+3 \beta)}} t+a_{4}, \\
& h=\frac{\sqrt{3} a_{6} a_{8} \sqrt{\beta} \sqrt{\gamma}}{\sqrt{a_{4}^{2}(\alpha+3 \beta)}} x+a_{6} y+\frac{a_{6} a_{8} \alpha \sqrt{\gamma}}{\sqrt{3 a_{4}^{2} \beta(\alpha+3 \beta)}} t+a_{8} .
\end{aligned}
$$

Figure 1 show the profile of $u_{1}$ and its density plots.

Note that the lump solution in (7) are analytic if the parameter satisfy $a_{4} \beta(\alpha+3 \beta) \neq 0$ and $\alpha \gamma<0$. We find that at any given time $t$, the above lump 

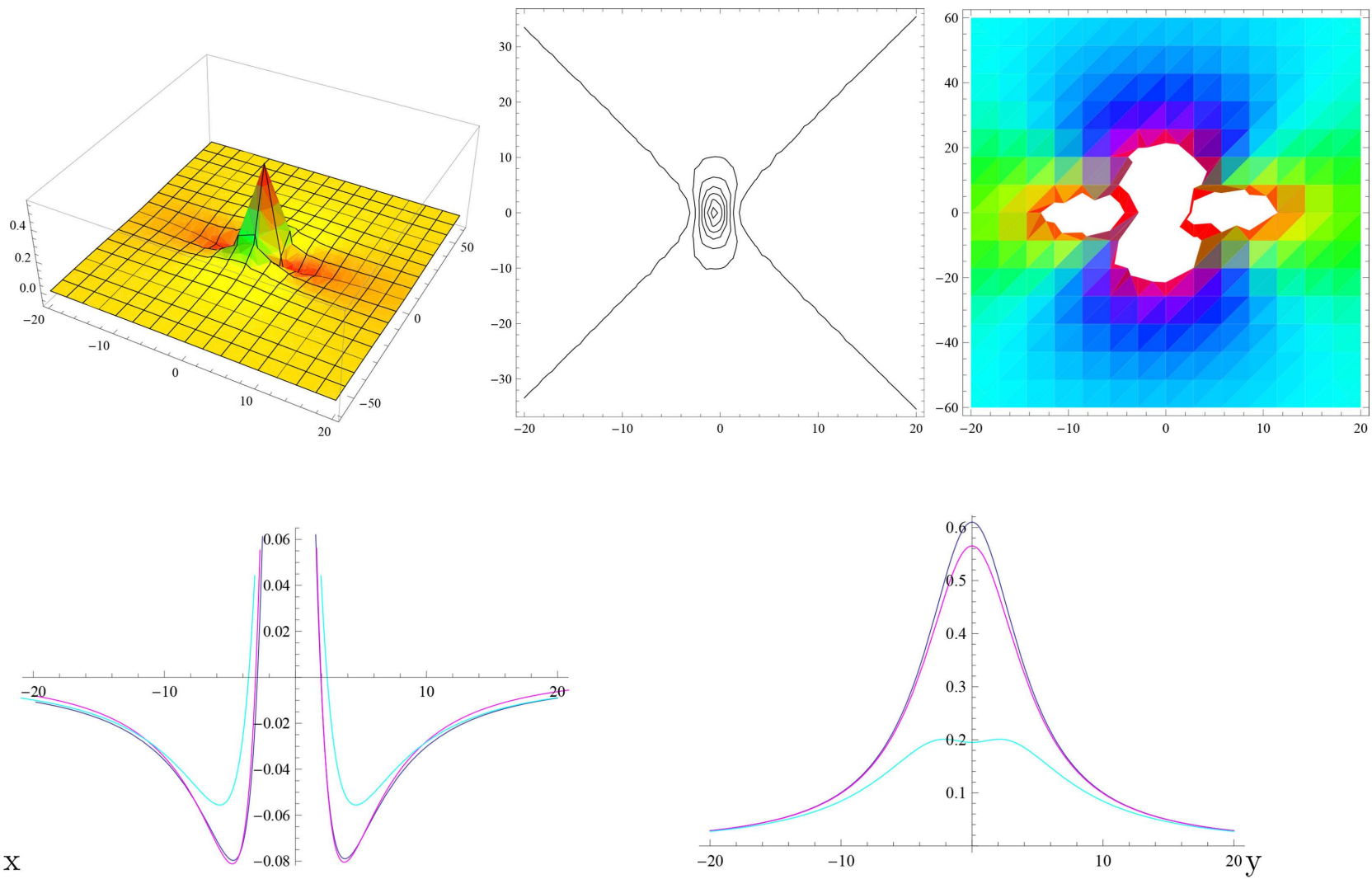

Figure 1. The profiles of $u_{1}(x, y, t)$ with $t=0$, density plot and $x, y$-curves with $a_{4}=1, a_{6}=1, a_{8}=1, \alpha=-2, \beta=1, \gamma=1$.

solutions $u \rightarrow 0$ if the corresponding sum of squares $g^{2}+h^{2} \rightarrow \infty$.

Case 2:

$$
\begin{gathered}
a_{1}=\frac{3 a_{3} \beta}{\alpha}, a_{2}=-\frac{a_{6} a_{5} \alpha}{3 a_{3} \beta}, a_{3}=-\frac{a_{6} \alpha \sqrt{\gamma}}{\sqrt{3} \sqrt{\beta} \sqrt{\alpha+3 \beta}}, a_{4}=a_{4}, a_{5}=a_{5}, a_{6}=a_{6}, \\
a_{7}=\frac{a_{1}^{2} \alpha+a_{5}^{2} \alpha-3 a_{1} a_{3} \beta}{3 a_{5} \beta}, a_{8}=a_{8}, a_{9}=-\frac{3 \alpha \beta\left(a_{5}^{2}(\alpha+3 \beta)+3 a_{6}^{2} \beta \gamma\right)}{(\alpha+3 \beta)^{2}},
\end{gathered}
$$

where $a_{4}, a_{5}, a_{6}, a_{8}$ are arbitrary constants, and all these constants are satisfied with the condition as follows:

$$
\gamma \beta \neq 0, \alpha+3 \beta \neq 0,3 \alpha \beta\left(a_{5}^{2}(\alpha+3 \beta)+3 a_{6}^{2} \beta \gamma\right)<0 .
$$

This set leads to a kind of positive quadratic function solutions to the bilinear $\mathrm{KP}-\mathrm{BBM}$ equation in (3)

$$
\begin{aligned}
f= & {\left[a_{8}+a_{6} y+a_{5}\left(x+\frac{t \alpha}{3 \beta}\right)\right]^{2}-\frac{3 \alpha \beta\left(a_{5}^{2}(\alpha+3 \beta)+3 a_{6}^{2} \beta \gamma\right)}{(\alpha+3 \beta)^{2}} } \\
& +\frac{\left[\sqrt{3} a_{5} y(\alpha+3 \beta)+3 a_{4} \sqrt{\beta \gamma(\alpha+3 \beta)}-\sqrt{3} a_{6}(t \alpha+3 x \beta) \gamma\right]^{2}}{9 \beta \gamma(\alpha+3 \beta)} .
\end{aligned}
$$

Analogously, we obtain the following lump solution to Equation (1) 


$$
\begin{aligned}
& u_{2}(x, y, t) \\
& =\left[-\frac{8\left(3 a_{5} a_{8} \beta(\alpha+3 \beta)+a_{5}^{2}(\alpha+3 \beta)(t \alpha+3 x \beta)-3 \sqrt{3} a_{4} a_{6} \beta^{\frac{3}{2}} \sqrt{(\alpha+3 \beta) \gamma}+3 a_{6}^{2} \beta(t \alpha+3 x \beta) \gamma\right)^{2}}{9 f^{2} \beta^{2}(\alpha+3 \beta)^{2}}\right] \\
& +\left[4\left(a_{5}^{2}+\frac{3 a_{6}^{2} \beta \gamma}{\alpha+3 \beta}\right)\right] \frac{1}{f}
\end{aligned}
$$

where the function $f$ is defined by (9).

Case 3:

$$
\begin{aligned}
& a_{1}=a_{1}, a_{2}=\frac{a_{4} a_{6}}{a_{8}}, a_{3}=\frac{a_{1} \alpha}{3 \beta}, a_{4}=a_{4}, a_{5}=a_{5}, a_{6}=\frac{a_{1} \sqrt{\alpha+3 \beta}}{\sqrt{3} \sqrt{\gamma} \sqrt{\beta}}, \\
& a_{7}=\frac{a_{5} \alpha}{3 \beta}, a_{8}=-\frac{a_{1} a_{4}}{a_{5}}, a_{9}=-\frac{3\left(a_{1}^{2}+a_{5}^{2}\right) \alpha \beta}{\alpha+3 \beta},
\end{aligned}
$$

where $a_{1}, a_{4}, a_{5}$ are arbitrary constants, and all these constants are satisfied with $a_{5} a_{8} \beta \gamma \neq 0, a_{2}^{2} a_{5}-2 a_{1} a_{2} a_{6}-a_{6}^{2} a_{5} \neq 0$ and $\frac{\alpha \beta}{\alpha+3 \beta}<0$.

This leads to

$$
\begin{aligned}
f= & {\left[a_{1}\left(x+\frac{t \alpha}{3 \beta}\right)-\frac{a_{5} y \sqrt{\alpha+3 \beta}}{\sqrt{3 \beta \gamma}}+a_{4}\right]^{2} } \\
& +\left(a_{5} x+\frac{a_{1} y \sqrt{\alpha+3 \beta}}{\sqrt{3} \sqrt{\gamma} \sqrt{\beta}}+\frac{a_{5} t \alpha}{3 \beta}-\frac{a_{1} a_{4}}{a_{5}}\right)^{2}-\frac{3\left(a_{1}^{2}+a_{5}^{2}\right) \alpha \beta}{\alpha+3 \beta} .
\end{aligned}
$$

Analogously, we obtain the following lump solution to Equation (1)

$$
u_{3}(x, y, t)=-\frac{36 a_{5}^{2} \beta^{2}(\alpha+3 \beta) \gamma\left[6 \sqrt{3} a_{4} a_{5} y \beta^{\frac{3}{2}} \sqrt{\gamma}(\alpha+3 \beta)^{\frac{3}{2}}-9 a_{4}^{2} \beta^{2}(\alpha+3 \beta) \gamma+a_{5}^{2}(-3 p+q)\right]}{-6 \sqrt{3} a_{4} a_{5} y \beta^{\frac{3}{2}}(\alpha+3 \beta)^{\frac{3}{2}} \sqrt{\gamma}+9 a_{4}^{2} \beta^{2}(\alpha+3 \beta) \gamma+a_{5}^{2}(3 p+q)},
$$

where

$$
\begin{aligned}
& p=y^{2} \beta(\alpha+3 \beta)^{2}, \\
& q=t^{2} \alpha^{2}(\alpha+3 \beta)+6 t x \alpha \beta(\alpha+3 \beta)+9 \beta^{2}\left[-3 \alpha \beta+x^{2}(\alpha+3 \beta)\right] \gamma .
\end{aligned}
$$

\section{Case 4:}

$$
\begin{aligned}
& a_{1}=-\frac{3 a_{6} a_{7} \beta}{a_{2} \alpha}, a_{2}=a_{2}, a_{3}=\frac{a_{1}^{2} \alpha+a_{5}^{2} \alpha-3 a_{5} a_{7} \beta}{3 a_{1} \beta}, a_{4}=\frac{a_{2} a_{8}}{a_{6}}, a_{5}=\frac{3 a_{7} \beta}{\alpha}, \\
& a_{6}=a_{6}, a_{7}=\frac{a_{2} \alpha \sqrt{\gamma}}{\sqrt{3 \beta} \sqrt{\alpha+3 \beta}}, a_{8}=a_{8}, a_{9}=-\frac{9\left(a_{2}^{2}+a_{6}^{2}\right) \alpha \beta^{2} \gamma}{(\alpha+3 \beta)^{2}},
\end{aligned}
$$

where $a_{2}, a_{6}, a_{8}$ are arbitrary constants, and all these constants are satisfied with $\gamma \alpha<0$ and $a_{1} a_{2} a_{6}(\alpha+3 \beta) \alpha \beta \neq 0$.

This leads to 


$$
f=\left(a_{8}^{2}+\frac{a_{2}^{2} a_{8}^{2}}{a_{6}^{2}}\right)+\frac{2\left(a_{2}^{2}+a_{6}^{2}\right) a_{8} y}{a_{6}}+\frac{\left(a_{2}^{2}+a_{6}^{2}\right)(3 p+q)}{3 \beta(\alpha+3 \beta)^{2}} .
$$

Analogously, we obtain the following lump solution to Equation (1)

$$
\begin{aligned}
& u_{4}(x, y, t) \\
& =-\frac{36 a_{6}^{2} \beta^{2}(\alpha+3 \beta) \gamma\left[-3 a_{8}^{2} \beta(\alpha+3 \beta)^{2}-6 a_{6} a_{8} y \beta(\alpha+3 \beta)^{2}+a_{6}^{2}(-3 p+q)\right]}{\left[3 a_{8}^{2} \beta(\alpha+3 \beta)^{2}+6 a_{6} a_{8} y \beta(\alpha+3 \beta)^{2}+a_{6}^{2}(3 p+q)\right]^{2}},
\end{aligned}
$$

where the functions $p, q$ is defined by (13).

Figure 2 show the profile of $u_{4}$ and its density plots.

Case 5:

$$
\begin{gathered}
a_{1}=\frac{3 a_{5} a_{7} a_{8} \beta}{2 a_{4} a_{5} \alpha-3 a_{4} a_{7} \beta}, a_{2}=-\frac{3 a_{7}^{2} a_{8} \beta(\alpha+3 \beta)}{a_{4} a_{6} \alpha^{2} \gamma}, a_{3}=\frac{a_{7} a_{8}}{a_{4}}, a_{4}=a_{4}, a_{5}=\frac{3 a_{7} \beta}{\alpha}, \\
a_{6}=\frac{\sqrt{3} a_{7} a_{8} \sqrt{\beta} \sqrt{\alpha+3 \beta}}{a_{4} \alpha \sqrt{\gamma}}, a_{7}=a_{7}, a_{8}=a_{8}, a_{9}=-\frac{27 a_{7}^{2}\left(a_{4}^{2}+a_{8}^{2}\right) \beta^{3}}{a_{4}^{2} \alpha(\alpha+3 \beta)},
\end{gathered}
$$

where $a_{4}, a_{7}, a_{8}$ are arbitrary constants, and all these constants are satisfied with $(\beta+3 \alpha) \gamma \alpha a_{4} a_{6} \neq 0,2 a_{4} a_{5} \alpha-3 \beta a_{4} a_{7} \neq 0$ and $\frac{\beta^{3}}{\alpha^{2}+3 \alpha \beta}<0$,

$$
\begin{aligned}
f= & -\frac{27 a_{7}^{2}\left(a_{4}^{2}+a_{8}^{2}\right) \beta^{3}}{a_{4}^{2} \alpha(\alpha+3 \beta)}+\frac{\left[a_{4}^{2} \alpha+a_{7} a_{8}(t \alpha+3 x \beta)-\frac{\sqrt{3} a_{4} a_{7} y \sqrt{\beta} \sqrt{\alpha+3 \beta}}{\sqrt{\gamma}}\right]^{2}}{a_{4}^{2} \alpha^{2}} \\
& +\left[a_{8}+a_{7}\left(t+\frac{3 x \beta}{\alpha}\right)+\frac{\sqrt{3} a_{7} a_{8} y \sqrt{\beta} \sqrt{\alpha+3 \beta}}{a_{4} \alpha \sqrt{\gamma}}\right]^{2} .
\end{aligned}
$$

Analogously, we obtain the following lump solution to Equation (1)

$u_{5}(x, y, t)$

$$
=\frac{36 a_{7}^{2} \beta\left(-2\left(a_{7} a_{8}^{2}(t \alpha+3 x \beta)+a_{4}^{2}\left(2 a_{8} \alpha+a_{7} t \alpha+3 a_{7} x \beta\right)\right)^{2}+a_{4}^{2}\left(a_{4}^{2}+a_{8}^{2}\right) \alpha^{2} f\right)}{a_{4}^{2} \alpha^{4} f^{2}},
$$

where the function $f$ is defined by (17).

\section{Interaction Solutions}

\subsection{Lump-Kink Solutions}

In this section, we will study the interaction between a lump and a stripe of the KP-BBM Equation (1). For search the interaction between rational solution and solitary wave solution, we turn the above function $f(x, y, t)$ into the following new form

$$
\begin{aligned}
f= & \left(a_{1} x+a_{2} y+a_{3} t+a_{4}\right)^{2}+\left(a_{5} x+a_{6} y+a_{7} t+a_{8}\right)^{2} \\
& +\exp \left(a_{9} x+a_{10} y+a_{11} t+a_{12}\right)+a_{13},
\end{aligned}
$$




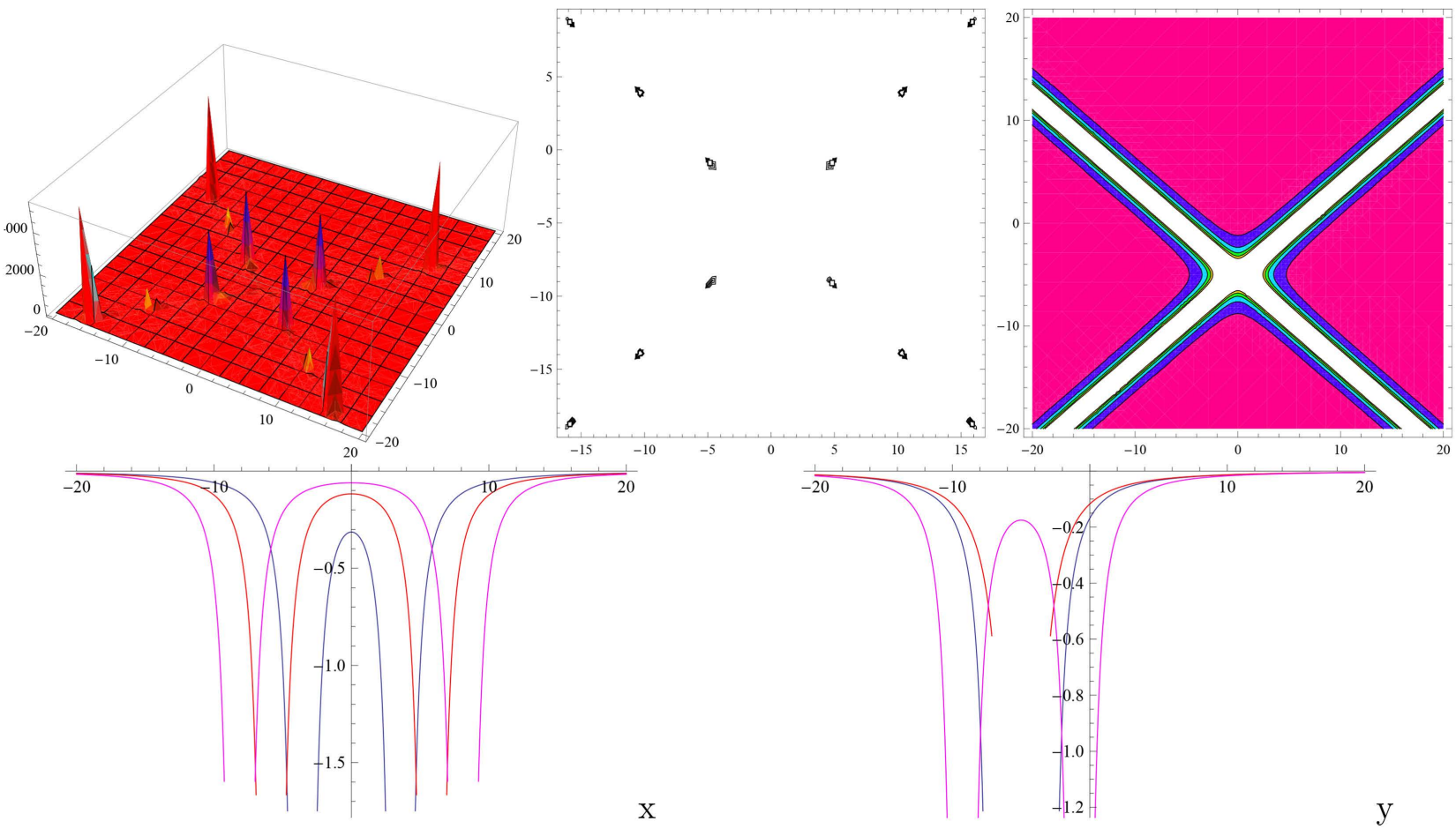

Figure 2. The profiles of $u_{4}(x, y, t)$ with $t=0$, density plot and $x$-curves with $a_{2}=1, a_{6}=1, a_{8}=5, \gamma=\beta=1, \gamma=-1$.

where $a_{i}(i=1, \cdots, 13)$ are real constants to be determined and $a_{13}>0$.

For Equation (1), substituting (19) into the corresponding bilinear form (3), direct Mathematica symbolic computations generate the following set of solutions for the parameters:

$$
\begin{aligned}
& a_{1}=-\frac{a_{5} a_{7}}{a_{3}}, a_{9}=0, \gamma=0, \beta=\frac{2}{3}, \alpha=-2, \\
& a_{i}=a_{i}(i=2,3,4,5,6,7,8,10,11,12,13),
\end{aligned}
$$

where $a_{3} \neq 0$. Then the exact interaction solution of $u$ is expressed as follows:

$$
\begin{aligned}
u_{1}= & 2(\ln f)_{x x} \\
= & 2\left[-\frac{\left(a_{9} \exp \left(a_{12}+a_{11} t+a_{9} x+a_{10} y\right)+2 a_{1}\left(a_{4}+a_{3} t+a_{1} x+a_{2} y\right)+2 a_{5}\left(a_{8}+a_{7} t+a_{5} x+a_{6} y\right)\right)^{2}}{\left(a_{13}+\exp \left(a_{12}+a_{11} t+a_{9} x+a_{10} y\right)+\left(a_{4}+a_{3} t+a_{1} x+a_{2} y\right)^{2}+\left(a_{8}+a_{7} t+a_{5} x+a_{6} y\right)^{2}\right)^{2}}\right. \\
& \left.+\frac{2 a_{1}^{2}+2 a_{5}^{2}+a_{9}^{2} \exp \left(a_{12}+a_{11} t+a_{9} x+a_{10} y\right)}{a_{13}+\exp \left(a_{12}+a_{11} t+a_{9} x+a_{10} y\right)+\left(a_{4}+a_{3} t+a_{1} x+a_{2} y\right)^{2}+\left(a_{8}+a_{7} t+a_{5} x+a_{6} y\right)^{2}}\right] .
\end{aligned}
$$

To obtain the collision phenomenon, $a_{3}^{2}+a_{7}^{2}+a_{11}^{2} \neq 0$ is necessary. So the asymptotic behavior of $u$ can be obtained, when

$$
\lim _{t \rightarrow \infty} u(x, t, y)=0 .
$$

The asymptotic behavior shows that the lump is finally submerged drowned or swallowed up by the stripe along with the change of time. Figure 3 exhibit the interaction between the lump soliton and kink soliton. The interaction between two solitary waves is inelastic. 

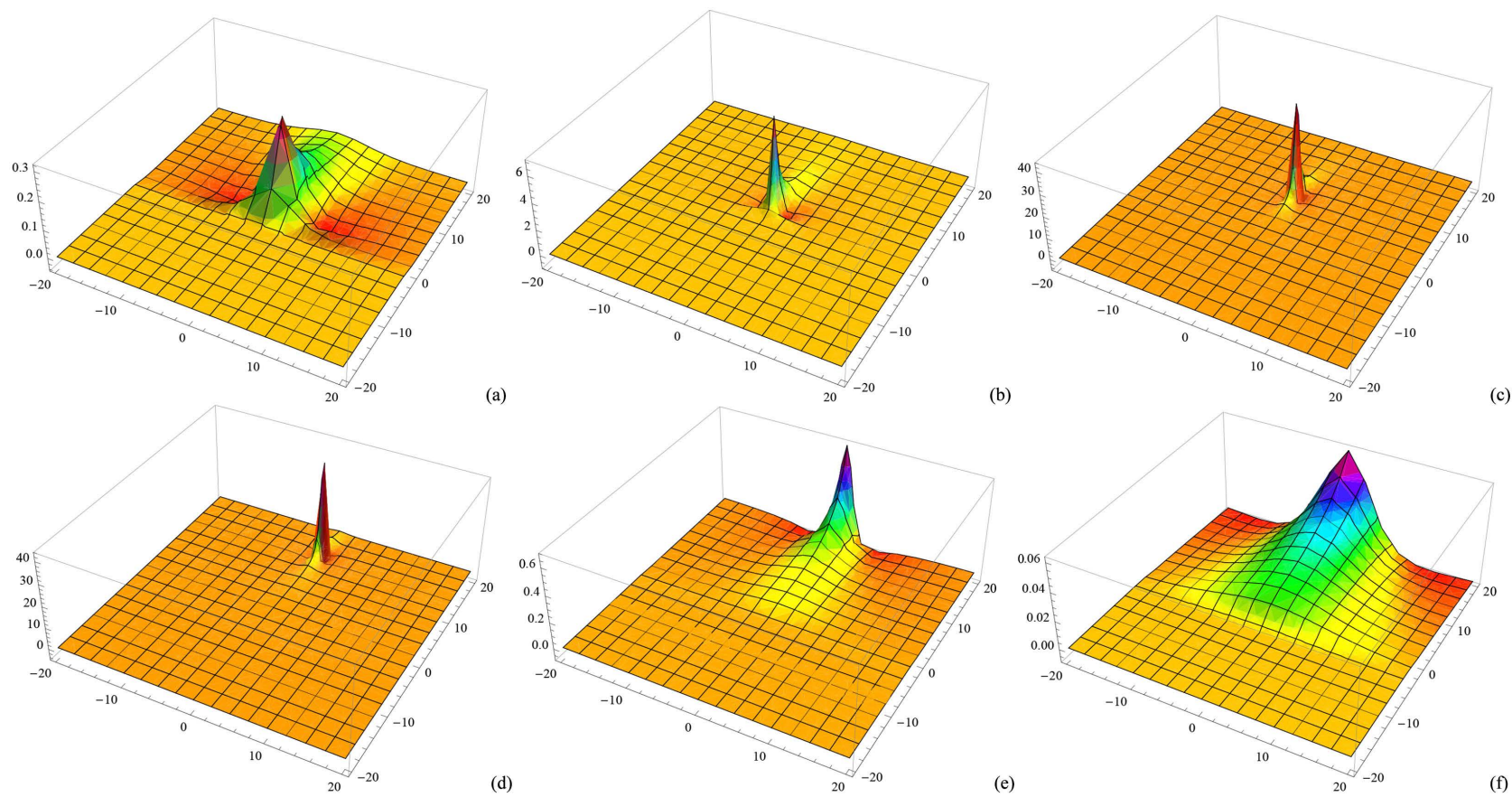

Figure 3. The interaction solution Equation $(21)$ for $u_{1}(x, y, t)$ with $a_{2}=-1, a_{3}=2, a_{4}=0, a_{5}=-\frac{5}{2}, a_{6}=-1, a_{7}=2$, $a_{8}=0, a_{10}=-3, a_{11}=-\frac{1}{3}, a_{12}=5, a_{13}=1$ when $t=3 \quad(\mathrm{a})(\mathrm{d}), t=0 \quad(\mathrm{~b})(\mathrm{e}), t=-3 \quad(\mathrm{c})(\mathrm{f})$.

\subsection{Lump-Soliton Solutions}

In the following, we compute interaction solutions between lumps and solitons to the KP-BBM Equation (1). It is combined functions of positive quadratic functions and hyperbolic cosine, and then we explored nonlinear phenomenon. We suppose

$$
\begin{aligned}
f= & \left(a_{1} x+a_{2} y+a_{3} t+a_{4}\right)^{2}+\left(a_{5} x+a_{6} y+a_{7} t+a_{8}\right)^{2} \\
& +\cosh \left(a_{9} x+a_{10} y+a_{11} t+a_{12}\right)+a_{13},
\end{aligned}
$$

where $a_{i}(i=1, \cdots, 13)$ are real constants to be determined and $a_{13}>0$. Substituting (22) into the Hirota bilinear form (3), direct symbol calculation results in a kind of solutions:

$$
\begin{aligned}
& a_{1}=\frac{4 a_{2} a_{6} \gamma}{a_{7}(-2+3 \beta)}, a_{2}=-\frac{a_{3} a_{6}}{a_{7}}, a_{3}=\frac{a_{7}}{\sqrt{3}}, \\
& a_{4}=0, a_{5}=0, a_{6}=a_{6}, a_{7}=a_{7}, a_{8}=a_{8}, \\
& a_{9}=\frac{\sqrt{-2+3 \beta}}{\sqrt{2 \beta}}, a_{10}=0, a_{11}=0, \alpha=-2, \\
& a_{12}=a_{12}, a_{13}=\frac{64 a_{6}^{4} \beta \gamma^{2}}{3 a_{7}^{2}(-2+3 \beta)^{3}} .
\end{aligned}
$$

Then we can obtain the soliton-lump soliton to Equation (1) with the transformation $u_{2}=2(\ln f)_{x x}$, where 


$$
\begin{aligned}
f= & \left(a_{8}+a_{7} t+a_{6} y\right)^{2}+\frac{64 a_{6}^{4} \beta \gamma^{2}}{3 a_{7}^{2}(-2+3 \beta)^{3}} \\
& +\frac{\left[a_{6} a_{7} y(2-3 \beta)+a_{7}^{2} t(-2+3 \beta)-4 a_{6}^{2} x \gamma\right]^{2}}{3 a_{7}^{2}(2-3 \beta)^{2}}+\cosh \left[a_{12}+\frac{x \sqrt{-2+3 \beta}}{\sqrt{2 \beta}}\right] .
\end{aligned}
$$

According to the Ref. [45], we can calculate the general formula of the original coordinates of lumps.

$$
\left(\frac{a_{2} a_{9} t-a_{4} a_{7} t-a_{5} a_{7}}{a_{1} a_{7}-a_{2} a_{6}},-\frac{a_{1} a_{9} t-a_{4} a_{6} t-a_{5} a_{6}}{a_{1} a_{7}-a_{2} a_{6}}\right)
$$

where $a_{1} a_{7}-a_{2} a_{6} \neq 0$. Then, substituting $a_{5}=0$ into formula (25). From formula (23), we know that the initial velocities in $x$ direction and $y$ direction of lump are $v_{x}=-\frac{a_{4} a_{7}-a_{2} a_{9}}{a_{1} a_{7}-a_{2} a_{6}}$ and $v_{y}=-\frac{a_{1} a_{9}-a_{4} a_{6}}{a_{1} a_{7}-a_{2} a_{6}}$. But in the collision, the lump and the solitary wave will exchange the energy, which will result in that the lump and the solitary wave will not be moving in the original trajectories or moving at the same speeds [46].

To illustrate the interaction phenomena between a lump and a stripe, we select the following parameters:

$$
a_{6}=-3, a_{7}=5, a_{8}=\frac{1}{2}, a_{12}=\frac{3}{5}, \beta=2, \gamma=1 .
$$

Figure 4 and Figure 5 show the profile of $u_{2}(x, y, t)$ and its contour plot with the parameters (26).

\section{Breather-Wave Solutions}

To construct the breather-wave solutions [47] [48], we assume that

$$
\left\{\begin{array}{l}
f=\exp \left(-p_{1} \mu\right)+\delta_{1} \cos (p \psi)+\delta_{2} \exp \left(p_{1} \mu\right), \\
\mu=x+a_{1} y+b_{1} t \\
\psi=x+a_{2} y+b_{2} t .
\end{array}\right.
$$

where $\mu$ and $\psi$ are the linear functions of $x, y, t$, while $p, a_{i}, b_{i}(i=1,2)$ and $p_{1}$ are real constants to be determined. To get the following results, we substitute the expression (27) into Bilinear Form (3) and eliminate the coefficients of $\exp \left(p_{1} \mu\right), \exp \left(-p_{1} \mu\right), \cos (p \psi)$.

$$
\begin{aligned}
e q[1]= & -2 p^{2} \delta^{2}\left(1+b_{2}+\alpha-3 b_{2} \beta-4 b_{2} p^{2} \beta+a_{2}^{2} \gamma\right), \\
e q[2]= & 8 p_{1}^{2} \delta_{2}\left(1+b_{1}+\alpha-3 b_{1} \beta+4 b_{1} p_{1}^{2} \beta+a_{1}^{2} \gamma\right), \\
e q[3]= & 2 p^{2} \delta_{1}^{2}\left(-1-b_{2}-\alpha+3 b_{2} \beta+4 b_{2} p^{2} \beta-a_{2}^{2} \gamma\right), \\
e q[4]= & 2 \delta_{1}\left(b_{2} p^{4} \beta+p_{1}^{2}\left(1+b_{1}+\alpha-3 b_{1} \beta+b_{1} p_{1}^{2} \beta+a_{1}^{2} \gamma\right)\right. \\
& \left.-p^{2}\left(1+b_{2}+\alpha-3 b_{2} \beta+3 b_{1} p_{1}^{2} \beta+3 b_{2} p_{1}^{2} \beta+a_{2}^{2} \gamma\right)\right), \\
e q[5]= & -2 p p_{1} \delta_{1}\left[b_{1}\left(1-3 \beta-p^{2} \beta+3 p_{1}^{2} \beta\right)\right] \\
& +b_{2}\left(1-3 \beta-3 p^{2} \beta+p_{1}^{2} \beta\right)+2\left(1+\alpha+a_{1} a_{2} \gamma\right) .
\end{aligned}
$$



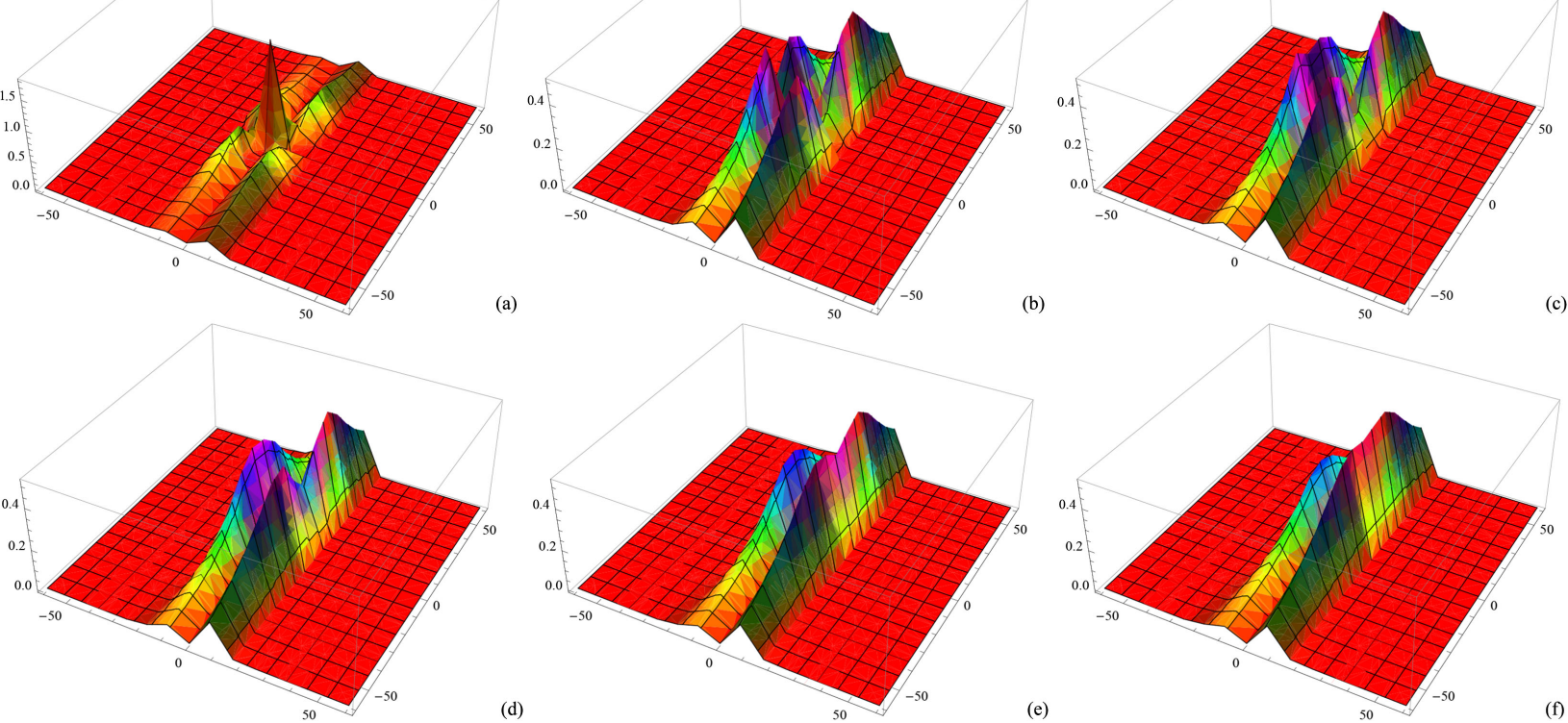

Figure 4. Profiles of $u_{2}(x, y, t)$ with the parameters (26) at times $t=0$ (a), $t=2$ (b), $t=4$ (c), $t=7$ (d), $t=10$ (e), $t=12$ (f).

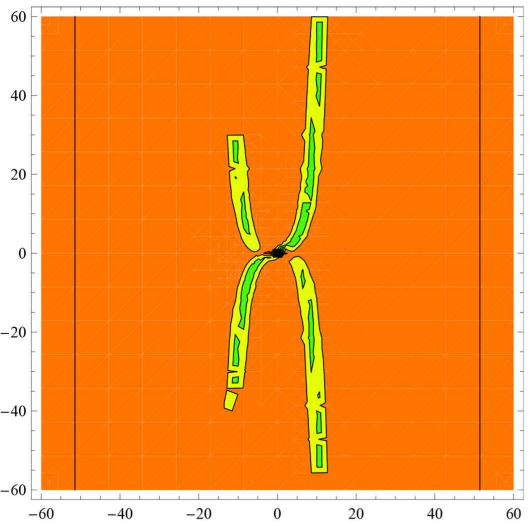

(a)

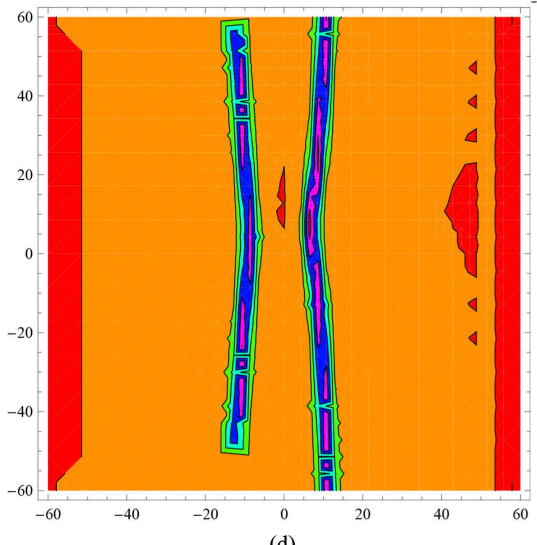

(d)

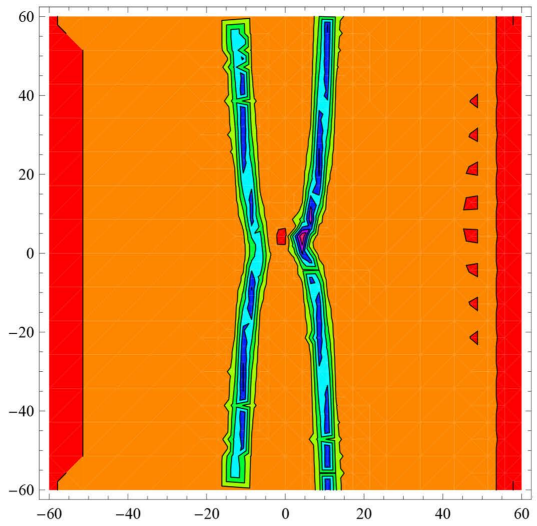

(b)

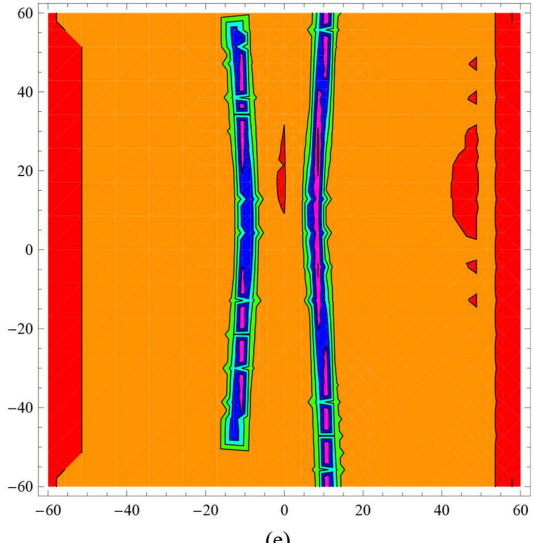

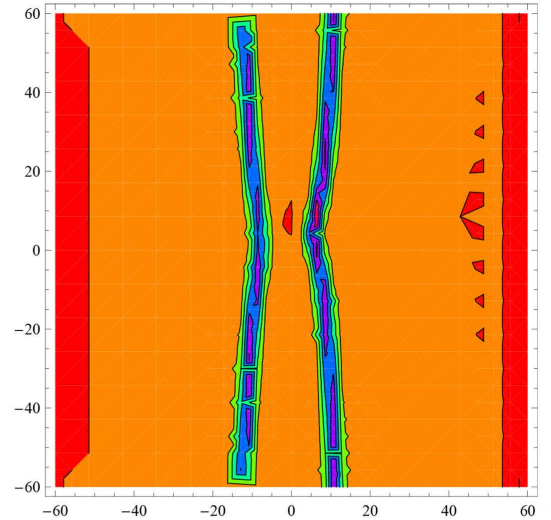

(c)

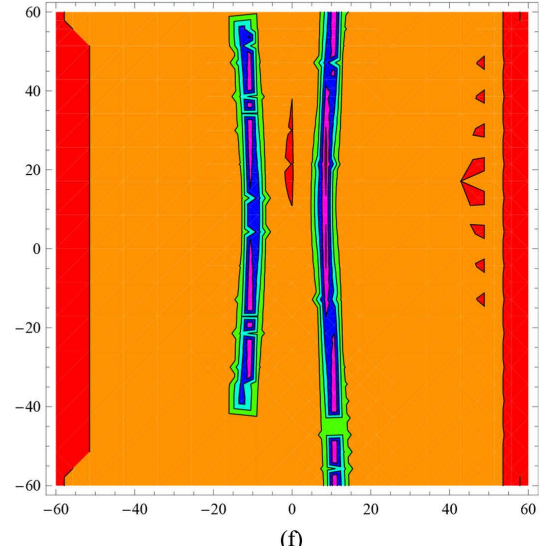

(f)

Figure 5. Contour plot of the $u_{2}(x, y, t)$ with the parameters $a_{6}=-3, a_{7}=5, a_{8}=\frac{1}{2}, a_{12}=\frac{3}{5}, \beta=2, \gamma=1$ when $t=0$ (a), $t=2$ (b), $t=4 \quad$ (c), $t=7 \quad$ (d), $t=10 \quad$ (e), $t=12 \quad$ (f). 
Then, we get the following restricted condition:

$$
\begin{aligned}
& b_{1}=-\frac{1+\alpha+a_{1}^{2} \gamma}{1-3 \beta+4 p_{1}^{2} \beta}, b_{2}=\frac{1+\alpha+a_{2}^{2} \gamma}{-1+3 \beta+4 p^{2} \beta}, a_{1}=a_{2}, \\
& p=\frac{p_{1} \sqrt{-1+3 \beta} \sqrt{1+\alpha+a_{1}^{2} \gamma}}{\sqrt{1+\alpha-3 \alpha \beta+a_{2}^{2} \gamma+\beta\left(-3-4 a_{1}^{2} p_{1}^{2} \gamma+a_{2}^{2}\left(-3 \gamma+4 p_{1}^{2} \gamma\right)\right)}} .
\end{aligned}
$$

and the expression (27) can be rewritten as

$$
\begin{aligned}
f(x, y, t)= & 2 \sqrt{\delta_{2}} \cosh \left[p_{1}\left(x+a_{2} y-\frac{1+\alpha+a_{1}^{2} \gamma}{1-3 \beta+4 p_{1}^{2} \beta}\right)+\frac{1}{2} \ln \delta_{2}\right] \\
& +\delta_{1} \cos \left[p\left(x+a_{2} y-\frac{t\left(1+\alpha+a_{2}^{2} \gamma\right)}{1-3 \beta-4 p_{1}^{2} \beta}\right)\right] .
\end{aligned}
$$

When we take $p=p_{1}$, lead to

$$
u(x, y, t)=\frac{2 p_{1}^{2} \sinh \left(p_{1} \mu+\frac{1}{2} \ln \delta_{2}\right)}{\left[2 \sqrt{\delta_{2}} \cosh \left(p_{1} \mu+\frac{1}{2} \ln \delta_{2}\right)+\delta_{1} \cos \left(p_{1} \psi\right)\right]^{2}} .
$$

\section{Conclusions}

In this paper, through Hirota transformation and Mathematica symbolic computation, we have presented five kinds of lump solutions and two classes of interaction phenomena to the KP-BBM Equation (1). Firstly, the analyticity and localization of the resulting solutions are ensured by some determinant conditions, and a sub-class of abundant lump solution includes different choices of the parameters and coefficient. Secondly, some contour plots with different determinant values which are sequentially made to show that the corresponding lump solutions tend to zero when $x^{2}+y^{2} \rightarrow \infty$. Thirdly, we explored two classes of interaction phenomenon in the literature. It showed in this work illustrate that the exponential-algebraic wave solution is unstable. At last, we obtain the breather-wave.

Solitons always have a connection between amplitude and width, so that it is independent. It is a different shape or profile. As we can see from the picture, lump solutions don't have such connection between amplitude and widths, and the profile shape can be quite random. Therefore, in the shape and formation lump solution process, the three-dimensional solitons solutions are more free than one-dimensional ones.

\section{Acknowledgements}

This work is supported by the National Natural Science Foundation of China (11661060,11571008), Natural Science Foundation of Inner Mongolia Autonomous Region of China (2018LH01013). 


\section{Conflicts of Interest}

The authors declare no conflicts of interest regarding the publication of this paper.

\section{References}

[1] Baskonus, H.M. (2016) New Acoustic Wave Behaviors to the Davey-Stewartson Equation with Power-Law Nonlinearity Arising in Fluid Dynamics. Nonlinear Dynamics, 86, 177-183. https://doi.org/10.1007/s11071-016-2880-4

[2] Baskonus, H.M. (2017) New Complex and Hyperbolic Function Solutions to the Generalized Double Combined Sinh-Cosh-Gordon Equation. AIP Conference Proceedings, 1798, 1-9(020018). https://doi.org/10.1063/1.4972610

[3] Cattani, C., Sulaiman, T.A., Baskonus, H.M., et al. (2018) On the Soliton Solutions to the Nizhnik-Novikov-Veselov and the Drinfel'd-Sokolov Systems. Optical and Quantum Electronics, 50, 138. https://doi.org/10.1007/s11082-018-1406-3

[4] Cattani, C., Sulaiman, T.A., Baskonus, H.M., et al. (2018) Solitons in an Inhomogeneous Murnaghan's Rod. European Physical Journal Plus, 133, 1-12. https://doi.org/10.1140/epjp/i2018-12085-y

[5] Sun, Y., Tian, B., Xie, X.Y., et al. (2017) Rogue Waves and Lump Solitons for a (3+1)-Dimensional B-Type Kadomtsev-Petviashvili Equation in Fluid Dynamics. Waves in Random and Complex Media, 28, 1-9.

[6] Villarroel, J., Prada, J. and Estvez, P.G. (2009) Dynamics of Lump Solutions in a 2+1 NLS Equation. Studies in Applied Mathematics, 122, 395-410. https://doi.org/10.1111/j.1467-9590.2009.00440.x

[7] Lü, J.Q., Bilige, S.D. and ChaoLu, T.M. (2018) The Study of Lump Solution and Interaction Phenomenon to (2+1)-Dimensional Generalized Fifth-Order KdV Equation. Nonlinear Dynamics, 91, 1669-1676.

[8] Kaup, D.J. (1981) The Lump Solutions and the Bäcklund Transformation for the Three-Dimensional Three-Wave Resonant Interaction. Journal of Mathematical Physics, 22, 1176-1181. https://doi.org/10.1063/1.525042

[9] Gilson, C.R. and Nimmo, J.J.C. (1990) Lump Solutions of the BKP Equation. Physics Letters A, 147, 472-476. https://doi.org/10.1016/0375-9601(90)90609-R

[10] Quintero, J.R. (2002) Existence and Analyticity of Lump Solutions for Generalized Benney-Luke Equations. Revista Colombiana de Matem'aticas, 36, 71-95.

[11] Singh, N. and Stepanyants, Y. (2016) Obliquely Propagating Skew KP Lumps. Wave Motion, 64, 92-102. https://doi.org/10.1016/j.wavemoti.2016.03.005

[12] Ma, W.X. (2015) Lump Solutions to the Kadomtsev-Petviashvili Equation. Physics Letters A, 379, 1975-1978. https://doi.org/10.1016/j.physleta.2015.06.061

[13] Ma, W.X. and Zhou, Y. (2018) Lump Solutions to Nonlinear Partial Differential Equations via Hirota Bilinear Forms. Journal of Differential Equations, 264, 2633-2659. https://doi.org/10.1016/j.jde.2017.10.033

[14] Zhang, H.Q. and Ma, W.X. (2016) Lump Solutions to the (2+1)-Dimensional Sawada-Kotera Equation. Nonlinear Dynamics, 87, 1-6.

[15] Zhang, H.Q. and Ma, W.X. (2017) Mixed Lump-Kink Solutions to the KP Equation. Computers and Mathematics with Applications, 74, 1399-1405. https://doi.org/10.1016/j.camwa.2017.06.034

[16] Ma, W.X., Qin, Z. and Lü, X. (2016) Lump Solutions to Dimensionally Reduced p-gKP and p-gBKP Equations. Nonlinear Dynamics, 84, 923-931. 
https://doi.org/10.1007/s11071-015-2539-6

[17] Lü, X., Chen, S.T. and Ma, W.X. (2016) Constructing Lump Solutions to a Generalized Kadomtsev-Petviashvili-Boussinesq Equation. Nonlinear Dynamics, 86, 1-12. https://doi.org/10.1007/s11071-016-2905-z

[18] Ma, W.X., Zhou, Y. and Dougherty, R. (2016) Lump-Type Solutions to Nonlinear Differential Equations Derived from Generalized Bilinear Equations. International Journal of Modern Physics B, 30, Article ID: 1640018. https://doi.org/10.1142/S021797921640018X

[19] Zhang, J.B. and Ma, W.X. (2017) Mixed Lump-Kink Solutions to the BKP Equation. Computers and Mathematics with Applications, 74, 591-596.

https://doi.org/10.1016/j.camwa.2017.05.010

[20] Gao, L.N., Zi, Y.Y., Yin, Y.H., Ma, W.X. and Lü, X. (2017) Bäcklund Transformation, Multiple Wave Solutions and Lump Solutions to a (3+1)-Dimensional Nonlinear Evolution Equation. Nonlinear Dynamics, 89, 2233-2240.

https://doi.org/10.1007/s11071-017-3581-3

[21] Yang, J.Y. and Ma, W.X. (2017) Abundant Lump-Type Solutions of the Jimbo-Miwa Equation in (3+1)-Dimensions. Computers and Mathematics with Applications, 52, 24-31. https://doi.org/10.1016/j.camwa.2016.11.007

[22] Yang, J.Y., Ma, W.X. and Qin, Z.Y. (2017) Lump and Lump-Soliton Solutions to the (2+1)-Dimensional Ito Equation. Analysis and Mathematical Physics, 1, 1-10.

[23] Lü, X. and Ma, W.X. (2016) Study of Lump Dynamics Based on a Dimensionally Reduced Hirota Bilinear Equation. Nonlinear Dynamics, 85, 1217-1222. https://doi.org/10.1007/s11071-016-2755-8

[24] Lü, J.Q. and Bilige, S.D. (2018) Diversity of Interaction Solutions to the (3+1)-Dimensional Kadomtsev-Petviashvili-Boussinesq-Like Equation. Modern Physics Letters B, 32, Article ID: 1850311.

[25] Chen, S.T. and Ma, W.X. (2018) Lump Solutions to a Generalized BogoyavlenskyKonopelchenko Equation. Frontiers of Mathematics in China, 13, 525-534. https://doi.org/10.1007/s11464-018-0694-Z

[26] Ma, W.X., Yong, X. and Zhang, H.Q. (2018) Diversity of Interaction Solutions to the (2+1)-Dimensional Ito Equation. Computers and Mathematics with Applications, 75, 289-295. https://doi.org/10.1016/j.camwa.2017.09.013

[27] Zhang, R.F., Bilige, S.D., et al. (2018) Interaction Phenomenon to Dimensionally Reduced p-gBKP Equation. Modern Physics Letters B, 32, Article ID: 1850074. https://doi.org/10.1142/S0217984918500744

[28] Ma, H.C. and Deng, A.P. (2016) Lump Solution of (2+1)-Dimensional Boussinesq Equation. Communications in Theoretical Physics, 65, 546-552. https://doi.org/10.1088/0253-6102/65/5/546

[29] Huang, L.L. and Chen, Y. (2017) Lump Solutions and Interaction Phenomenon for (2+1)-Dimensional Sawada-Kotera Equation. Communications in Theoretical Physics, 67, 473-478. https://doi.org/10.1088/0253-6102/67/5/473

[30] Wang, Y.H., Wang, H., Zhang, H.S. and ChaoLu, T.M. (2017) Exact Interaction Solutions of an Extended (2+1)-Dimensional Shallow Water Wave Equation. Communications in Theoretical Physics, 68, 165. https://doi.org/10.1088/0253-6102/68/2/165

[31] Sun, H.Q. and Chen, A.H. (2016) Lump and Lump-Kink Solutions of the (3+1)-Dimensional Jimbo-Miwa and Two Extended Jimbo-Miwa Equations. Applied Mathematics Letters, 68, 55-61. https://doi.org/10.1016/j.aml.2016.12.008 
[32] Liu, D.Y., Tian, B. and Xie, X.Y. (2017) Lump Solutions for the (3+1)-Dimensional Kadomtsev-Petviashvili Equation. Zeitschrift für Naturforschung A, 71, 1079-1173.

[33] Tang, Y., Tao, S. and Guan, Q. (2016) Lump Solitons and the Interaction Phenomena of Them for Two Classes of Nonlinear Evolution Equations. Computers and Mathematics with Applications, 72, 2334-2342. https://doi.org/10.1016/j.camwa.2016.08.027

[34] Zhao, Z., Chen, Y. and Han, B. (2017) Lump Soliton, Mixed Lump Stripe and Periodic Lump Solutions of a (2+1)-Dimensional Asymmetrical Nizhnik-Novikov-Veselov Equation. Modern Physics Letters B, 14, Article ID: 1750157. https://doi.org/10.1142/S0217984917501573

[35] Zhang, X. and Chen, Y. (2017) Rogue Wave and a Pair of Resonance Stripe Solitons to a Reduced (3+1)-Dimensional Jimbo-Miwa Equation. Communications in Nonlinear Science and Numerical Simulation, 52, 24-31. https://doi.org/10.1016/j.cnsns.2017.03.021

[36] Chen, M.D., Li, X., Wang, Y., et al. (2017) A Pair of Resonance Stripe Solitons and Lump Solutions to a Reduced (3+1)-Dimensional Nonlinear Evolution Equation. Communications in Theoretical Physics, 67, 595-600. https://doi.org/10.1088/0253-6102/67/6/595

[37] Zhang, Y., Dong, H.H., Zhang, X.E. and Yang, H.W. (2016) Rational Solutions and Lump Solutions to the Generalized (3+1)-Dimensional Shallow Water-Like Equation. Computers and Mathematics with Applications, 73, 246-252. https://doi.org/10.1016/j.camwa.2016.11.009

[38] Lv, J.Q. and Bilige, S.D. (2017) Lump Solutions of a (2+1)-Dimensional bSK Equation. Nonlinear Dynamics, 90, 2119-2124. https://doi.org/10.1007/s11071-017-3788-3

[39] Wu, X.Y., Tian, B., Chai, H.P., et al. (2017) Rogue Waves and Lump Solutions for a (3+1)-Dimensional Generalized B-Type Kadomtsev-Petviashvili Equation in Fluid Mechanics. Modern Physics Letters B, 31, Article ID: 1750122. https://doi.org/10.1142/S0217984917501226

[40] Wazwaz, A.M. (2005) Exact Solutions of Compact and Noncompact Structures for the KP-BBM Equation. Applied Mathematics and Computation, 169, 700-712. https://doi.org/10.1016/j.amc.2004.09.061

[41] Wazwaz, A.M. (2008) The Extended Tanh Method for New Compact and Noncompact Solutions for the KP-BBM and the ZK-BBM Equations. Chaos Solitons and Fractals, 38, 1505-1516. https://doi.org/10.1016/j.chaos.2007.01.135

[42] Saut, J.C. and Tzvetkov, N. (2004) Global Well-Posedness for the KP-BBM Equations. Applied Mathematics Research Express, 1, 1-16. https://doi.org/10.1155/S1687120004010718

[43] Alam, M.N. and Akbar, M.A. (2014) Exact Traveling Wave Solutions of the KP-BBM Equation by Using the New Approach of Generalized (G'/G)-Expansion Method. Springerplus, 3, 43. https://doi.org/10.1186/2193-1801-3-43

[44] Tang, S., Huang, X. and Huang, W. (2010) Bifurcations of Travelling Wave Solutions for the Generalized KP-BBM Equation. Applied Mathematics and Computation, 216, 2881-2890. https://doi.org/10.1016/j.amc.2010.03.139

[45] Fokas, A.S., Pelinovsky, D.E. and Sulaem, C. (2001) Interaction of Lumps with a Line Soliton for the DSII Equation. Journal of Physics D, 152-153, 189-198. https://doi.org/10.1016/S0167-2789(01)00170-1

[46] Tang, Y., Tao, S., Zhou, M., et al. (2017) Interaction Solutions between Lump and 
Other Solitons of Two Classes of Nonlinear Evolution Equations. Nonlinear Dynamics, 89, 1-14. https://doi.org/10.1007/s11071-017-3462-9

[47] Tajiri, M. and Watanabe, Y. (1998) Breather Solutions to the Focusing Nonlinear Schrödinger Equation. Physical Review E Statistical Physics Plasmas Fluids and Related Interdisciplinary Topics, 57, 3510-3519.

https://doi.org/10.1103/PhysRevE.57.3510

[48] Zhao, H.H., Zhao, X.J. and Hao, H.Q. (2016) Breather-to-Soliton Conversions and Nonlinear Wave Interactions in a Coupled Hirota System. Applied Mathematics Letters, 61, 8-12. https://doi.org/10.1016/j.aml.2016.04.016 